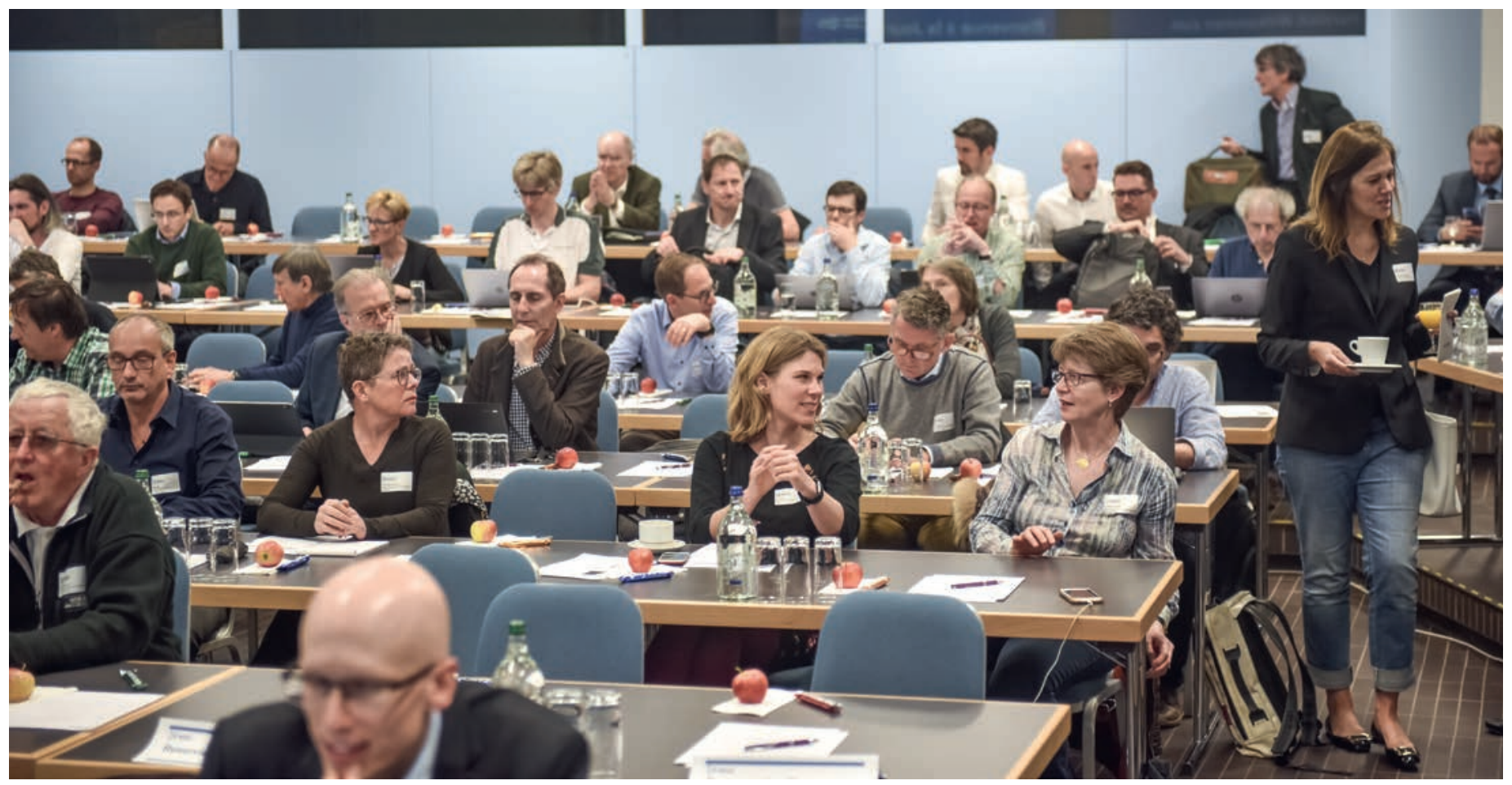

\title{
Wird der revidierte Arzttarif zum Amtstarif?
}

\section{Thomas Kessler ${ }^{\text {, Christian Oeschger }}{ }^{b}, K$ Kerstin Schutz ${ }^{c}$, Sabine Zehnder ${ }^{d}$, Chaim Hännie, Nora Wille ${ }^{f}$}

${ }^{a} \mathrm{FMH}$, Experte, Abteilung Ambulante Versorgung und Tarife; ${ }^{\mathrm{b}} \mathrm{FMH}$, Projektleiter, Abteilung Ambulante Versorgung und Tarife; ${ }^{c}$ FMH, Expertin,

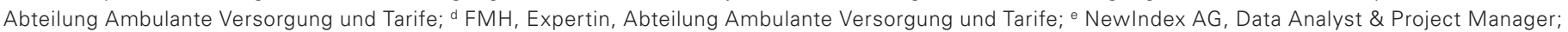
${ }^{\dagger} \mathrm{FMH}$, persönliche wissenschaftliche Mitarbeiterin des Präsidenten

Am 11. April 2019 fand in Bern unter dem Titel «Wird der revidierte Arzttarif zum Amtstarif?» der erste Tarifdelegierten-Tag der FMH dieses Jahres statt. An dieser Veranstaltung nahmen wiederum gegen 100 interessierte Tarifdelegierte der Fachgesellschaften und der kantonalen Ärztegesellschaften teil. Als Gastreferentin durften wir Frau Sandra Schneider, Leiterin der Abteilung Tarife und Grundlagen des BAG, begrüssen, die zum Thema «Nationale ambulante Tariforganisation aus Sicht des BAG» sprach.

\section{Tarifrevision im dynamischen politischen Umfeld}

Dr. med. Jürg Schlup, Präsident der FMH, referiert über die Tarifrevision im dynamischen politischen Umfeld. Einleitend ging Jürg Schlup auf das politische Umfeld der Tarifrevision ein, das von einer sehr hohen Dyna- mik geprägt ist. Ähnlich wie beim Brexit, bei dem zehn Tage vor dem Tarifdelegierten-Tag der weitere Verlauf noch in keinster Weise absehbar war, sind in der Tarifrevision verschiedenste Interessensgruppen aufgefordert, in einem gegebenen Zeitraum zu einem gemeinsamen Ergebnis zu kommen. Die von aussen vorgegebenen Bedingungen spielen dabei den Interes- 


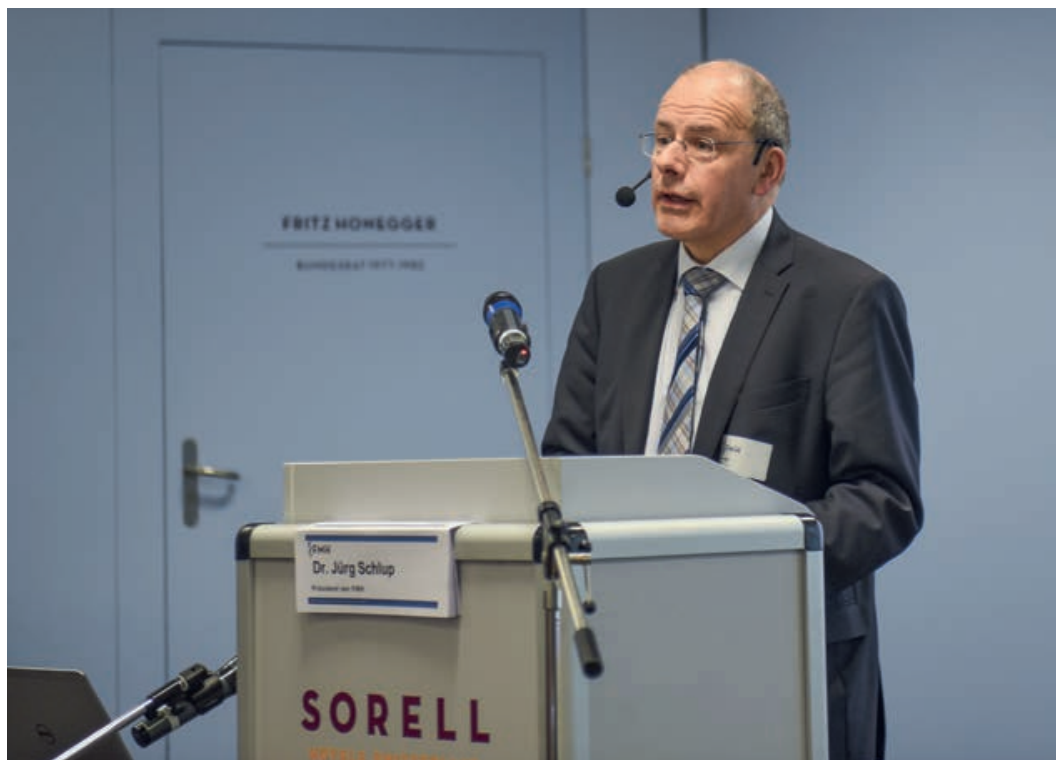

Dr. med. Jürg Schlup referiert über die Tarifrevision im dynamischen politischen Umfeld.

sen einiger Gruppen in die Hände - und wirken sich auf die Verhandlungsposition anderer Gruppen negativ aus. Seit dem letzten Tarifdelegierten-Tag nahm der politische Druck auf den Tarif weiter zu. Beispielhaft dafür führte Jürg Schlup aus, wie in der Kommunikation zur BAG-Bass-Studie die Ärzteschaft mit Falschaussagen diskreditiert wurde und Ärztelöhne ins falsche Licht gerückt wurden. Vielfach wurde hier auch suggeriert, die BAG-Bass-Studie erlaube Aussagen über die Sachgerechtigkeit des Tarifs, obwohl sie nicht einmal verschiedene Einkommensquellen differenzieren kann.

Der Tarif steht auch wegen der geplanten Einführung des Globalbudgets im Zentrum des politischen Interesses. Mit dem ersten Kostendämpfungspaket des Bundesrats liegt bereits eine Gesetzesvorlage vor, die einen Kostendeckel für den Arzttarif vorsieht, wenn auch versteckt. Weitere Vorstösse fordern ganz offen eine Budgetierung, so die vom Bundesrat im März angekündigte "Zielvorgabe», die «Kostenbremse» der CVP oder die Beschlüsse der SVP für ihr Parteiprogramm. Wo Kostendeckel ohne Leistungsdeckel versprochen werden, soll die Ärzteschaft die Differenz bezahlen und dafür braucht der Staat Zugriff auf den Arzttarif. Jürg Schlup dankte den Tarifdelegierten, dass sie trotz des schwierigen und unsicheren Umfelds die mühselige, aber extrem wichtige Arbeit leisten, die für eine erfolgreiche Tarifrevision unverzichtbar ist. Sie lösen den ärztlichen Anspruch, auch einen kritischen Patienten nicht aufzugeben - und die erreichten Meilensteine wie die verabschiedete Leistungsstruktur können sich sehen lassen. Die Tarifdelegierten schaffen mit hohem Einsatz gegen alle Widerstände die zentralen Voraussetzungen für die Tarifpartnerschaft und Tarifrevision - und damit die Chance, die Tarifautonomie zurückzugewinnen.

\section{Einleitung und tarifarische Neuigkeiten}

Dr. med. Urs Stoffel, Mitglied des Zentralvorstandes der FMH, informiert über die tarifarischen Neuigkeiten. Im Zentrum von Urs Stoffels Referat standen aktuelle Informationen zur gesundheitspolitischen Lage, Informationen zur nationalen ambulanten Tariforganisation aus Sicht des Bundesamtes für Gesundheit BAG, das Monitoring des 2. Tarifeingriffes - Resultate und Erkenntnisse -, der Stand der ambulanten Tarifrevision und die Einstimmung auf die bevorstehende interne Genehmigung des Tarifvorschlags.

Monitoring des Tarifeingriffs des Bundesrates: Zur Arbeitsgruppe «Umsetzung des Tarifeingriffes» des BAG teilte Urs Stoffel mit, dass das BAG die Tarifpartner am 26. Februar 2019 für eine gemeinsame Analyse und Interpretation des ersten Halbjahrs 2018 eingeladen hat. Jeder Tarifpartner konnte während 10 Minuten seine Analysen und Interpretation vorstellen. Das BAG hat sich bei der FMH ausdrücklich für die sehr detaillierten und sehr guten Analysen bedankt. Die Interpretationen des BAG decken sich in grossen Teilen mit den Analysen der FMH.

Tarifrevision TARDOC: Die Verhandlungen mit den Tarifpartnern werden in der ats-tms AG geführt (ohne Beteiligung von santésuisse). Urs Stoffel wies noch einmal darauf hin, dass das im Mai 2018 abgeschlossene Projekt TARCO nicht mit den laufenden Verhandlungen mit den Tarifpartnern gleichzusetzen ist. Die ausserordentliche Delegiertenversammlung vom 24. Oktober 2018 und die Ärztekammer vom 25. Oktober 2018 haben fast einstimmig die verhandelte ambulante Leistungsstruktur verabschiedet (1. Etappe). Damit war der Weg frei für die weiteren Verhandlungen rund um die Abrechnungs- und Anwendungsregeln (2. Etappe). Der gemeinsam verhandelte Tarif erhält die Bezeichnung TARDOC.

Zur FMH-internen Genehmigung teilte Urs Stoffel mit, dass eine ausserordentliche Delegiertenversammlung der FMH und kurz darauf die Ärztekammer Anfang Mai 2019 über den vorliegenden Tarifvorschlag entscheiden werden.

Zum Schluss teilte Urs Stoffel mit, dass, falls alle Tarifpartner der ats-tms dem Vorschlag zustimmen, das gesamte Paket der Tarifstruktur TARDOC Ende Juni 2019 dem Bundesrat eingereicht werden kann. Eine Genehmigung durch den Bundesrat ist aber nur möglich, wenn die Mehrheit der Tarifpartner den Tarifvor- 


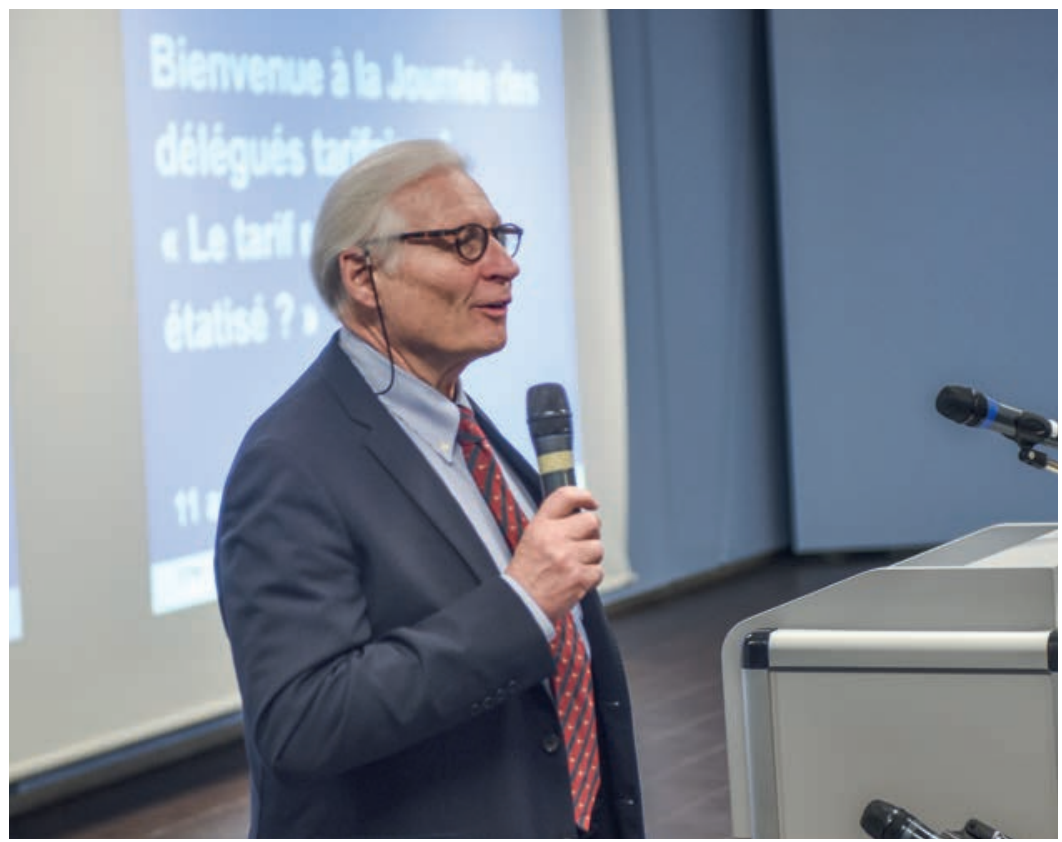

Dr. med. Urs Stoffel referiert über tarifarische Neuigkeiten.

schlag einreicht. Sollte das nicht der Fall sein, wird der Vorschlag vom Bundesrat geprüft, in eine Vernehmlassung gegeben, und letztlich kann der Bundesrat dann den Tarif für alle Tarifpartner verbindlich festlegen.

\section{Nationale ambulante Tariforganisation aus Sicht des BAG}

Sandra Schneider, stv. Leiterin des Direktionsbereiches Kranken- und Unfallversicherung und Leiterin der Abteilung Tarife und Grundlagen des BAG, referiert über

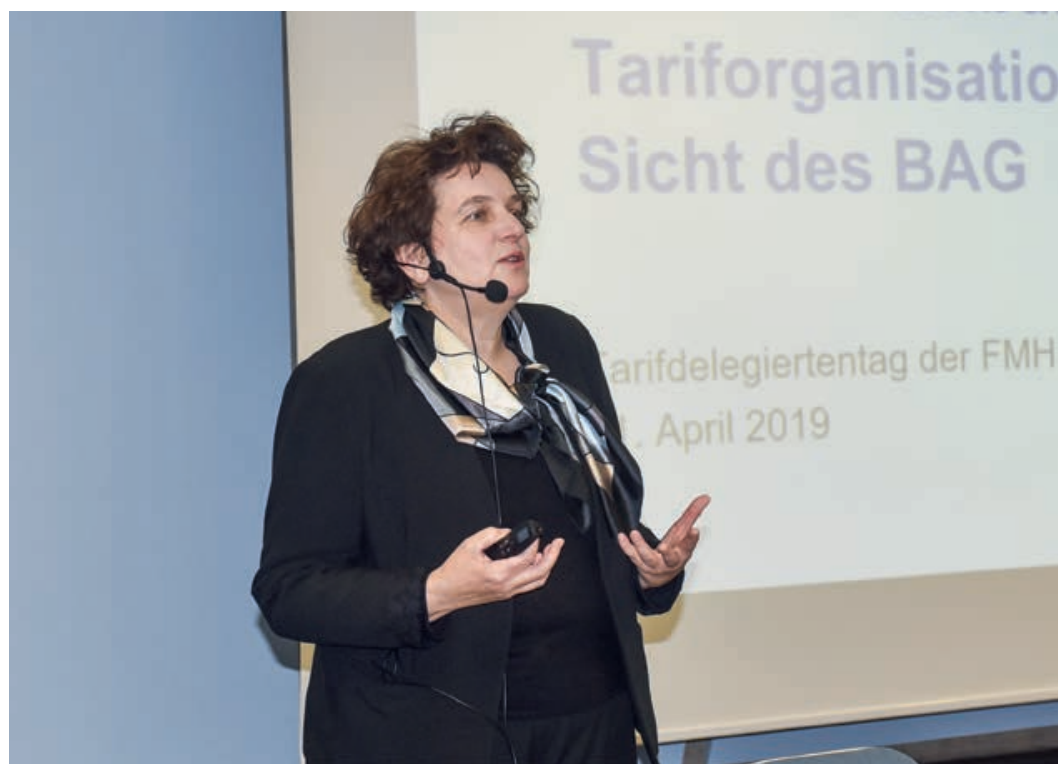

Sandra Schneider informiert über die nationale ambulante Tariforganisation aus Sicht des BAG. die nationale ambulante Tariforganisation aus Sicht des BAG.

Gemäss Art. 43 Absatz 4 KVG gilt: «Tarife und Preise werden in Verträgen zwischen Versicherern und Leistungserbringern (Tarifvertrag) vereinbart oder in den vom Gesetz bestimmten Fällen von der zuständigen Behörde festgesetzt.» Gemäss Art. 49 Absatz 2 KVG gilt im stationären Bereich, dass die Tarifpartner gemeinsam mit den Kantonen eine Organisation einsetzen, die für die Erarbeitung und Weiterentwicklung sowie die Anpassung und Pflege der Strukturen zuständig ist. Fehlt eine solche Organisation, so wird sie vom Bundesrat für die Tarifpartner verpflichtend eingesetzt. Im ambulanten Bereich fehlt bisher eine analoge gesetzliche Bestimmung.

Im Bereich der ambulanten ärztlichen Leistungen (TARMED) funktionierte die Tarifautonomie jedoch von Anfang an nicht wunschgemäss. Zudem gibt es neue bzw. veränderte Verhandlungssituationen (z.B. aufgrund der Abspaltung von curafutura vom Verband santésuisse). Um einen tarifstrukturlosen Zustand zu verhindern, musste der Bundesrat in diesem Bereich subsidiär eingreifen. Weil die einfache Gesellschaft TARMEDSuisse keine Entscheidungen mehr traf und de facto handlungsunfähig wurde, gründeten die Tarifpartner FMH, H+, curafutura und MTK am 18. Januar 2016 die Tariforganisation ats-tms AG.

santésuisse und der Spitalverband beteiligen sich zurzeit nicht an der ats-tms AG. Es fehlt damit im ambulanten Bereich eine Tariforganisation unter Beteiligung aller Tarifpartner. Nach Vorbild des stationären Bereichs soll nun auch im ambulanten Bereich eine Organisation eingesetzt werden, welche für die Erarbeitung und Weiterentwicklung sowie die Anpassung und Pflege von Tarifstrukturen zuständig ist. Die Verbände der Leistungserbringer und der Versicherer werden verpflichtet, auch im ambulanten Bereich eine Organisation einzusetzen, welche für die Erarbeitung und Weiterentwicklung sowie die Anpassung und Pflege der ambulanten Tarifstrukturen zuständig ist. Es wurde dazu eine Vernehmlassung gemacht, welche auf breite Zustimmung gestossen ist.

\section{Neues Analysetool der NewIndex AG für Tarifdelegierte}

Dr. Philip Moline und Chaim Hänni wiesen darauf hin, dass die ärzteeigene Datensammlung eine unverzichtbare Datengrundlage für die Ärzteorganisationen ist, insbesondere auch für die Arbeit der Tarifdelegierten. Die NewIndex hat zu diesem Zweck zusammen mit der Firma TMA ein neues Online-Analysetool für die Ärzteorganisationen und ihre Tarifdelegierten entwi- 


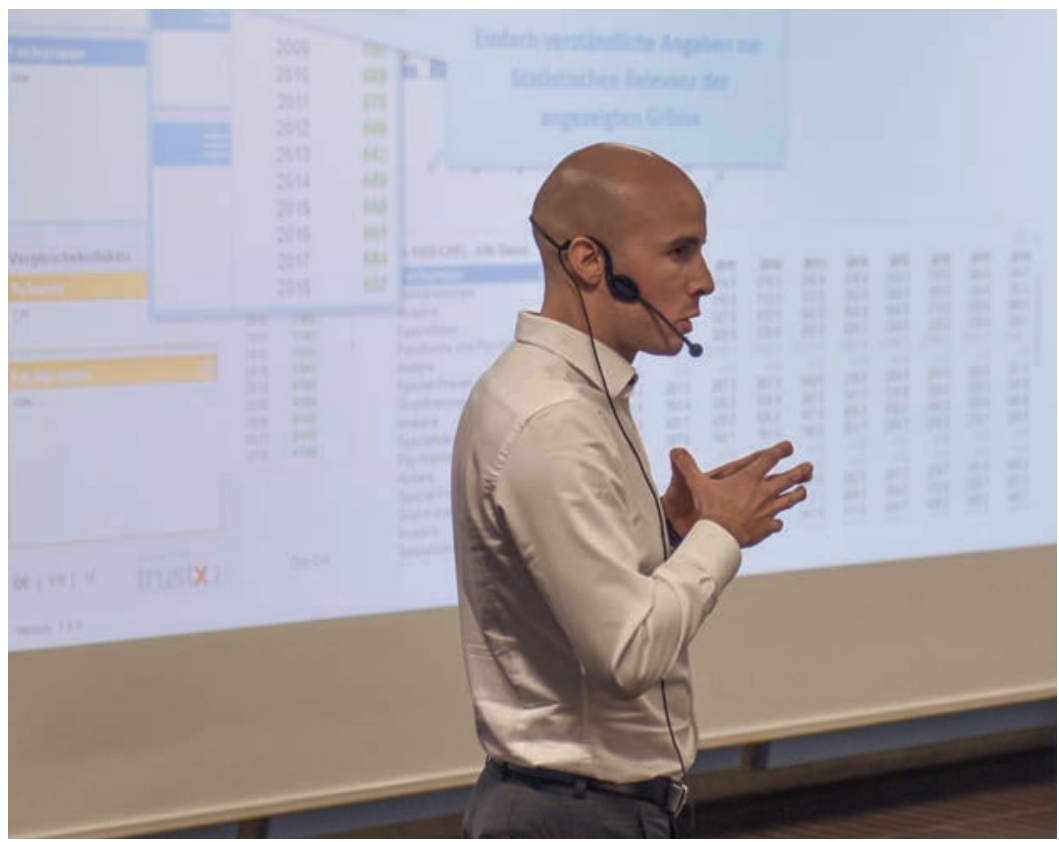

Chaim Hänni referiert über ein neues Analysetool der Newlndex AG für Tarifdelegierte.

ckelt. Es erlaubt ihnen, einfache Analysen ihrer wichtigsten Kennzahlen aus dem ärzteeigenen Datenpool selber vorzunehmen. Dabei wurde besonders Wert auf die Einfachheit der Bedienung und die logische Darstellung der Resultate gelegt. Es wird möglich sein, auch spezifische Auswertungsbedürfnisse zu aktuellen Themen aus den Ärzteorganisationen zu integrieren.

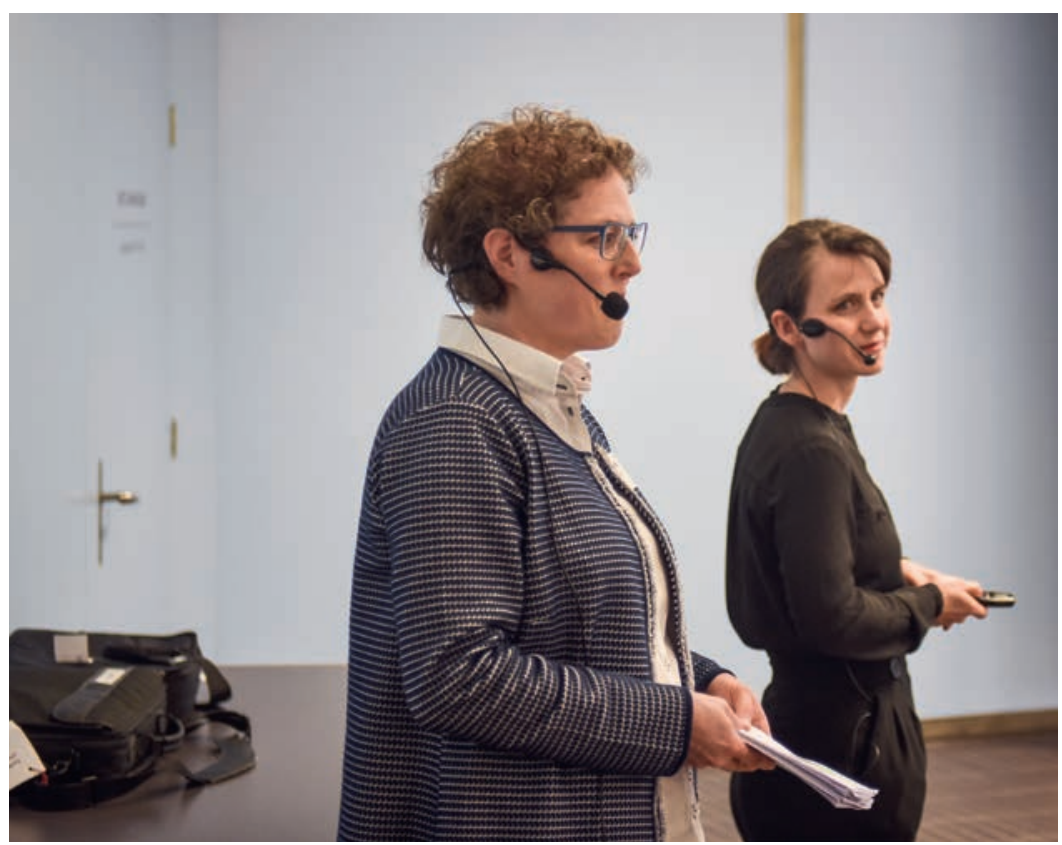

Kerstin Schutz und Sabine Zehnder referieren über das Monitoring des 2. Tarifeingriffs Resultate und Erkenntnisse.

\section{Monitoring 2. Tarifeingriff - Resultate und Erkenntnisse}

Kerstin Schutz und Sabine Zehnder informierten über das vom Bundesrat verordnete Monitoring des Tarifeingriffs.

Die FMH konnte dank der exzellenten Datenlage und in enger Zusammenarbeit mit der Ärztekasse und der NewIndex detaillierte und komplexe Analysen vornehmen und damit proaktiv voreiligen Fehlinterpretationen der Auswirkungen des Tarifeingriffs entgegenwirken.

Die FMH hat in all ihren Datenlieferungen und Analysen zuhanden des BAG als wichtige Botschaft immer wieder betont, dass Entwicklungen von einzelnen Positionen nicht isoliert betrachtet werden dürfen und diese Entwicklungen immer im Kontext zu den anderen Positionen interpretiert werden müssen. In den Begleittexten und Berichten wurden für die Ärzteschaft wichtige Hinweise auf mögliche Fehlinterpretationen und Hintergrundinformationen geliefert.

Neben Entwicklungen des Taxpunktvolumens hat das BAG auch Aussagen und Analysen zu Überschreitungsquoten der neu für alle Ärzte geltenden Limitationen gefordert. Auch diese Analysen zeigten, dass die Limitationen durch die Ärzteschaft eingehalten wurden.

\section{Ambulante Tarifrevision - der verhandelte Tarif steht!}

Christian Oeschger berichtet, dass in den vergangenen Wochen sämtliche Fachgesellschaften der FMH in Zusammenarbeit mit curafutura und der MTK das komplexe Regelwerk zur bereits im Oktober 2018 gemeinsam finalisierten Leistungsstruktur ausgearbeitet haben. In über 40 ganztägigen Workshops haben die Tarifdelegierten der Fachgesellschaften mit den Expertinnen und Experten sämtliche Tarifpositionen durchgearbeitet und für jede Position festgelegt, ob und wenn ja wo die Leistung limitiert werden kann. Ebenso haben die Verhandlungsdelegationen Kumulationseinschränkungen beschlossen.

Kerstin Schutz, Sabine Zehnder und Christian Oeschger legten den Fokus in ihrer Präsentation auf ebendiese Anwendungs- und Abrechnungsregeln. Dabei wurde auch aufgezeigt, wo die wesentlichen Änderungen in den Kostenmodellen, den generellen Interpretationen und auf Kapitelebene liegen.

Die Expertinnen und Experten der FMH haben die anwesenden Tarifdelegierten der kantonalen Ärztegesellschaften und der Fachgesellschaften auch darüber informiert, welche Simulationstools für die Entscheidungsfindung zur Verfügung stehen. In Zu- 

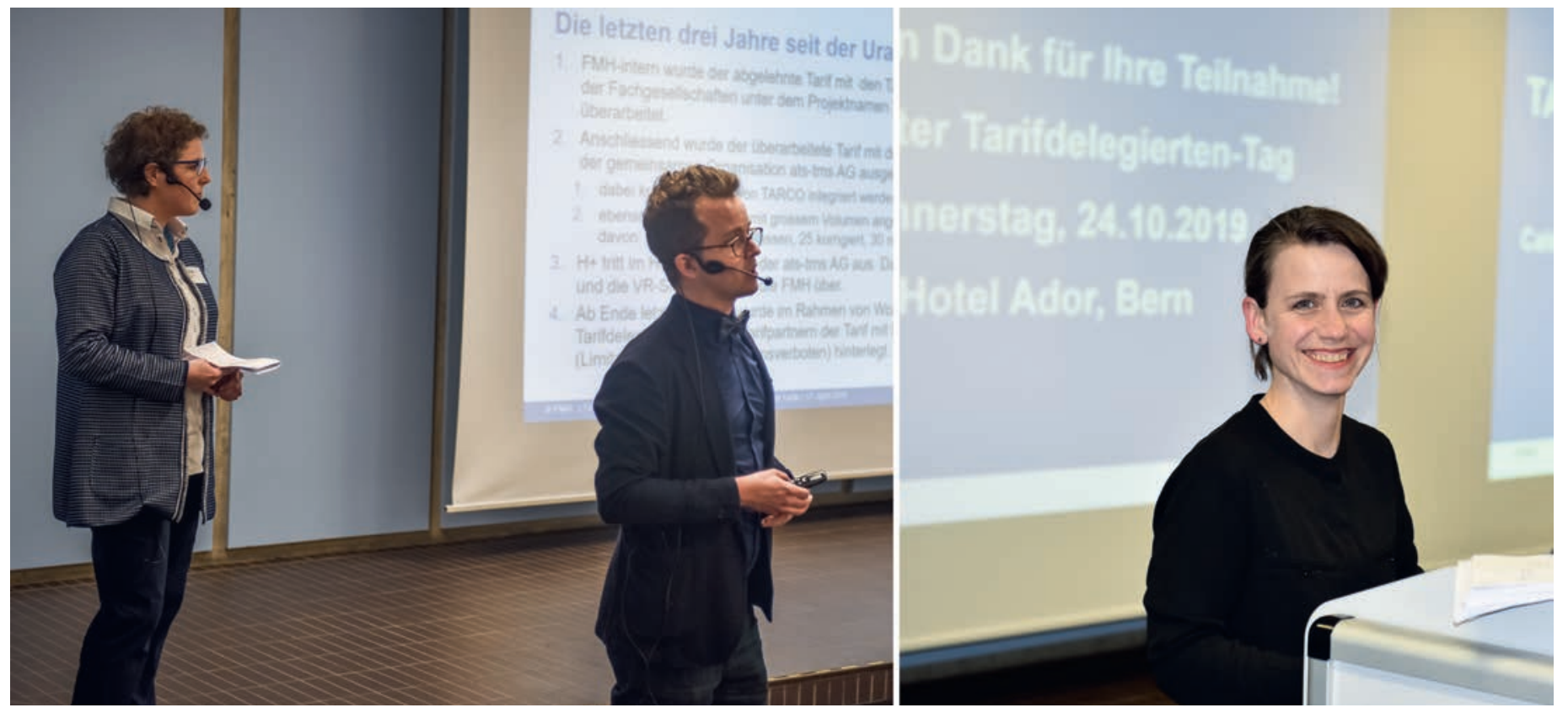

Christian Oeschger, Kerstin Schutz und Sabine Zehnder informieren über die tarifpartnerschaftliche ambulante Tarifrevision.

Korrespondenz: FMH, Abteilung Ambulante Versorgung und Tarife Baslerstrasse 47 CH-4600 Olten Tel. 0313591230 Fax 0313591238 tarife.ambulant[at]fmh.ch sammenarbeit mit der Ärztekasse und der NewIndex AG werden wiederum Volumenberechnungen sowie Warenkörbe analysiert, die es den Gesellschaften erlauben sollen, den Beschluss zur neuen Tarifstruktur zu fassen.

Weiterführende Informationen zum neuen Tarif TARDOC finden Sie auch in der Schweizerischen Ärztezeitung vom 24. April 2019.

\section{Zu guter Letzt}

Zum Abschluss zieht Urs Stoffel folgende Bilanz: Der Druck seitens Politik und Medien auf die Ärzteschaft nimmt weiter zu; unter anderem auch aufgrund des Massnahmenpaketes der Expertengruppe, der Auswirkungen des 2. Tarifeingriffs und der Situation im Bereich des Praxislabors. Die Bevölkerung wird durch den Prämiendruck in der Wahrnehmung sensibler. Die Gesundheitskosten werden daher auch zum Wahlkampfthema 2019. Nicht zuletzt sorgen die laufenden
Volksinitiativen zu den Prämien- und Gesundheitskosten dafür, dass dieses Thema weiterhin auf der Agenda bleibt.

Nutzen wir diese Chance und Möglichkeit und beweisen, dass die Ärzteschaft fähig ist, gemeinsam mit den Tarifpartnern einen revidierten Tarif zu verhandeln. Kämpfen wir gemeinsam und geschlossen für eine qualitativ hochstehende Gesundheitsversorgung mit adäquater und sachgerechter Abgeltung der Leistungen im Interesse unserer Patientinnen und Patienten.

Folien: Alle Unterlagen sowie die Gesamtpräsentation finden Sie auf der Website der FMH: www.fmh.ch $\rightarrow$ TARMED $\rightarrow$ Tarifdelegierten-Tag

Nächster Termin: Der nächste Tarifdelegierten-Tag findet Donnerstag, 24. Oktober 2019, im Hotel Ador in Bern statt. Bitte reservieren Sie sich den Termin! 